\title{
5'-AMP-Activated Protein Kinase Regulates Goat Sperm Functions via Energy Metabolism In Vitro
}

\author{
Zhendong Zhu Rongnan Li Gongzhen Ma Wenjing Bai Xiaoteng Fan \\ Yinghua Lv Jun Luo Wenxian Zeng \\ College of Animal Science and Technology, Northwest A\&F University, Shaanxi, China
}

\section{Key Words}

Acrosome Reaction • Capacitation • AMPK • Energy Metabolism • Sperm

\begin{abstract}
Background/Aims: ATP is essential for mammalian sperm to survive and maintain fertilizing capacity. AMP-activated protein kinase (AMPK) is a sensor of cellular energy status. The aims of the present study were to explore the localization of AMPK in goat sperm and to investigate whether and how AMPK regulates sperm functions in vitro. Methods: Sperm were treated with AMPK modulators (AICAR, metformin and Compound C) during incubation. Sperm motility was assessed with a computer-assisted spermatozoa analysis system (CASA). Membrane integrity, acrosome reaction and mitochondrial membrane potentials were detected by SYBR-14/PI, FITC-PNA and JC-1 staining, respectively. And the lactate content, ATP content, AMPK activity, activity of pyruvate kinase (PK) and lactate dehydrogenase (LDH) were also measured with the commercial assay kits. Immunofluorescence staining was used to analyze the distribution of PK, LDH, AMPK and phospho-Thr172-AMPK in sperm. The role of AMPK was further studied during induction of capacitation and acrosome reaction. Results: We found that AMPK $\alpha$ was localized in the entire acrosomal region, the midpiece and the flagellum, while the phosphoThr172-AMPK was distributed in the head, the midpiece and flagellum. Activation of AMPK by AICAR and metformin significantly improved sperm motility, membrane integrity and acrosome reaction, largely maintained sperm mitochondrial membrane potentials, lactate content and ATP content, and enhanced the activity of AMPK, PK and LDH, whereas inhibition by Compound $\mathrm{C}$ triggered the converse effects. Moreover, PK was localized in the acrosomal area and the midpiece, while LDH was distributed in the tail. Induction of capacitation and acrosome reaction led to AMPK phosphorylation. AMPK phosphorylation regulated the activity of energetic enzymes. Conclusion: This study for the first time provides evidence that AMPK governs goat sperm functions through energy metabolism in vitro. This finding will help to improve assisted reproductive techniques in goats and the other species.
\end{abstract}




\section{Introduction}

Mammalian sperm are specialized cells that require abundant ATP as energy source to chronically maintain motility. Capacitation is a process in which sperm undergo biochemical changes before fertilizing eggs [1]. The protein tyrosine phosphorylation is one of the most important intracellular signaling events regarded as a meaningful indicator for capacitation [2]. After capacitation, sperm display hyperactivation that is a special movement because of the increased flagellar bending with asymmetric waveform [3]. Hyperactivation is helpful to release of sperm from the oviduct reservoirs and to penetrate the extracellular matrix of the eggs [4]. When sperm approach the egg, they go through a process known as the acrosome reaction that removes plasma membrane in the area of acrosomal cap and exocytosing acrosomal content [5]. ATP is needed for both hyperactivation and acrosome reaction [6].

AMP-activated protein kinase (AMPK) is a key cellular sensor of energy status [7]. AMPK is a heterotrimeric protein composed of a catalytic $\alpha$ subunit and two regulatory submits $\beta$ and $\gamma$ [8]. Within the $\alpha$ subunit, a typical serine/threonine protein kinase domain is at the $\mathrm{N}$-terminus, while a regulatory domain at $\mathrm{C}$-terminus, which is activated by phosphorylation of the Thr172 residue that located at the critical activation loop of the $\alpha$ subunit [9-11]. Activation of AMPK speeds up ATP production via catabolic pathways to maintain cellular energy state [12].

Hurtado de Llera et al. reported that AMPK was expressed in boar sperm [13]. Subsequently, evidences have shown that AMPK was also present in stallion [14], chicken $[15]$ and human $[16,17]$ sperm. On one hand, AMPK activation enhanced sperm motility $[13,15-19]$, acrosome reaction $[15,19,20]$. On the other hand, AMPK inhibition led to a significant decrease in motility, mitochondria membrane potential and acrosome integrity during long-term storage of boar semen [21]. However, Shabani Nashtaei et al. demonstrated that AMPK inhibition did not affect membrane integrity in human sperm [17]. Moreover, Cordova et al. [14] reported that addition of neither AMPK activators nor AMPK inhibitors to the freezing extenders affected motility, membrane integrity and acrosome integrity in stallion sperm.

Dairy goat provides abundant meat, wool and milk. As such dairy goat has an important economic value in China. Artificial insemination is widely used in goat industry. Developing a strategy to improve sperm fertilization is of importance. To date, whether and how AMPK regulates goat sperm functions are unknown. Therefore, the present study was conducted to detect the localization of AMPK and phosphorylated Thr172 AMPK in goat sperm, and to elucidate the role of AMPK in sperm functions and its underlying mechanisms.

\section{Materials and Methods}

\section{Experiment design}

Experiment 1 was designed to identify the localization of AMPK and phospho-Thr172-AMPK, and to detect the effects of AMPK modulators (AICAR, metformin and Compound C) on sperm quality. Sperm were incubated with various concentrations of $\operatorname{AICAR}(0,2,4 \mathrm{mM})$, metformin (Met, 0, 0.5, 1, $1.5 \mathrm{mM}$ ) and Compound C $(0,15,30,45 \mu \mathrm{M})$ for $1 \mathrm{~h}$, respectively. We found that exposure of sperm to either $2 \mathrm{mM}$ of AICAR or $1 \mathrm{mM}$ of Met could maintain high values of sperm parameters, whereas exposure to $30 \mu \mathrm{M}$ for Compound C led to a decrease in the values of the parameters (for all online suppl. material, see www. karger.com/doi/10.1159/000491616, Suppl. Fig. 1). As sperm migration from the insemination location to fertilization site takes 1-2 $\mathrm{h}$ in the pigs and $4 \mathrm{~h}$ in the horse [22], effects of AMPK modulators on goat sperm parameters were further evaluated post $3 \mathrm{~h}$ of incubation. Sperm was exposed to either $2 \mathrm{mM}$ of AICAR or $1 \mathrm{mM}$ of Met, or $30 \mu \mathrm{M}$ of Compound C. At $3 \mathrm{~h}$ of incubation, we observed that it was also the case as $1 \mathrm{~h}$ of incubation. Therefore, these doses were used in the subsequent experiments.

Experiment 2 was to elucidate whether AMPK was related with sperm functions. The amount of phospho-Thr172-AMPK, AMPK activity, acrosome reaction and sperm parameters were detected during induction of capacitation and acrosome reaction in vitro. 


\section{Cellular Physiology Cell Physiol Biochem 2018;47:2420-2431 \begin{tabular}{l|l} 
and Biochemistry Published online: July 09, 2018 & $\begin{array}{l}\text { ( ) 2018 The Author(s). Published by S. Karger AG, Basel } \\
\text { www.karger.com/cpb }\end{array}$
\end{tabular} \\ Zhu et al.: AMPK is Involved in Goat Sperm Functions}

Experiment 3 was to elucidate the mechanism by which AMPK regulated the functions. Firstly, to test whether AMPK activity was associated with ATP generation, sperm were incubated with AMPK modulators for $3 \mathrm{~h}$ of incubation. ATP content, lactate content, activity of pyruvate kinase (PK) and lactate dehydrogenase (LDH), and phospho-Thr172-AMPK were measured. Secondly, to evaluate whether AMPK modulates capacitation and acrosome reaction via regulating energetic metabolism, lactate content, ATP content, PK and LDH were assessed after capacitation and acrosome reaction. Thirdly, to verify that AMPK regulates capacitation, sperm were incubated with AMPK modulators, the total number of sperm bound to zona pellucida was counted.

\section{Reagents and media}

All chemicals were purchased from Sigma (Shanghai, China), unless specified. 5-aminoimidazole4-carboxamide-1-beta-4-ribofuranoside (AICAR) was obtained from Beyotime Biotechnology (Nanjing, China). A23817 and Compound C were from Aladdin (Shanghai, China). Anti-p-Thr172-AMPK and anti- $\alpha$ tubulin, anti-pyruvate kinase (PK) and anti-LDH antibodies were from Santa Cruz Biotechnology (Santa Cruz, CA, USA), anti-AMPK was from Abcam (Shanghai, China), anti-phosphotyrosine (PTyr-100, 9411) was from Cell Signaling (Shanghai, China). Goat anti-rabbit IgG conjugated FITC or goat anti-mouse IgG conjugated $\mathrm{Cy} 3$, anti-mouse IgG conjugated horseradish peroxidase (HRP) and anti-rabbit IgG conjugated HRP were from Xi'An Zhuangzhi Biotechnology (Xian, China).

TCG extenders contained $250 \mathrm{mM}$ Tris [hydroxymethyl] aminomethane, $83 \mathrm{mM}$ of citric acid anhydrous and $69 \mathrm{mM}$ of $\mathrm{D}(+)$ glucose; $300 \mathrm{mOsm}, \mathrm{pH}=7$ [23]. Capacitation medium (CM) and Non-capacitation medium (NCM) were prepared according to Salmon et al. [24].

\section{Animals and semen collection}

All animals and experimental procedures were approved by the Northwest A\&F University Institutional Animal Care and Use Committee. Fifteen mature goats in the Xinong Saanen Dairy Goat Farm, China were used in this study. Buck were housed individually, maintained under natural daylight, fed basal diets and been free access to water. Semen was collected from each buck twice a week with an artificial vagina. Semen samples were pooled to avoid individual differences in this study. Fresh sperm were incubated in CM for up to $3 \mathrm{~h}$ at $38.5^{\circ} \mathrm{C}$ to induce capacitation, while sperm incubated with NCM as a control (non-capacitation condition). Acrosome reaction was induced by the addition of calcium ionophore A23187 (10 $\mu \mathrm{M})$

\section{Immunofluorescence}

Sperm samples were fixed with paraformaldehyde (4\%) for 5 min. The sperm were spread onto the slides, then permeabilized with $0.5 \%$ Triton X-100 in PBS. Samples were incubated overnight at $4{ }^{\circ} \mathrm{C}$ with 1:50-diluted anti-AMPK, anti-PK, anti-LDH or anti-phospho-Thr172-AMPK, then incubated with biotinylated goat anti-rabbit FITC-IgG or goat anti-mouse Cy3-IgG (1:200). Subsequently, sperm were incubated with 4',6'-diamidino-2-phenylindole (DAPI; $5 \mu \mathrm{g} / \mathrm{mL}$; Sigma-Aldrich). The presence of total AMPK, PK, LDH and phospho-Thr172-AMPK were examined under a fluorescence microscopy (Nikon, Japan). Negative control was performed at the same time without the primary antibody

\section{Western blotting}

The proteins were separated by 12\% SDS-PAGE and transferred onto nitrocellulose membrane (Millipore). Non-specific binding sites were blocked by incubation in Tris-buffered saline (TBS) containing $0.1 \%$ Tween-20 and 5\% dry non-fat milk. The membranes were immunoblotted with primary antibodies (anti-phospho-Thr172 AMPK (62 kDa), anti-total AMPK (62 kDa), anti-phosphotyrosine (PTyr-100) and anti$\alpha$-tubulin (55 kDa)) diluted in 5\% bovine serum albumin in TBS-Tween 0.1\% (1:1000 dilution), followed by incubation with HRP conjugated secondary (goat anti-rabbit antibody for phospho-Thr172AMPK and total AMPK, goat anti-mouse for $\alpha$-tubulin and anti-phosphotyrosine, 1:2000 dilution). The reagent for enhanced chemiluminescence (Bio-Rad) was used for detection and developed by X-ray film.

\section{Assessment of AMPK activity}

AMPK activity was assessed using a AMPK ELISA Kit (CK-E79152, China) according to the manufacturer's instruction. Briefly, sperm samples were mixed with HRP-Conjugate reagent in the Microelisa stripplate, following by addition of Chromogen Solution A and B. Subsequently, stop solution was added to each well. 


\section{Cellular Physiology Cell Physiol Biochem 2018;47:2420-2431 \begin{tabular}{l|l} 
and Biochemistry Published online: July 09, 2018 & $\begin{array}{l}\text { D } 2018 \text { The Author(s). Published by S. Karger AG, Basel } \\
\text { www.karger.com/cpb }\end{array}$
\end{tabular} \\ Zhu et al.: AMPK is Involved in Goat Sperm Functions}

Blank wells without sample and HRP-Conjugate reagent were set separately. The absorbance read was monitored at $450 \mathrm{~nm}$ using a spectrophotometer.

\section{Measurement of ATP concentration and lactate concentration}

ATP concentration was measured using a ATP Assay Kit (Beyotime Institute of Biotechnology, China). Briefly, samples were mixed luciferin/luciferase reagent in 96-well plates. The luminescence at integration $\times 1000$ ms was read using an Ascent Luminoskan luminometer (Thermo Scientific, Palm Beach, FL) with BPSE as a blank.

Lactate concentration was measured using a lactate detection kit (Nanjing Jiancheng, China), according to the manufacturer's instruction. Briefly, lactate concentration was measured by detecting the NADH formed consequently to lactate oxidation by LDH with a spectrophotometry at $340 \mathrm{~nm}$. BPSE was used as a blank.

\section{Detection of pyruvate kinase and LDH activity}

Activity of PK and LDH were detected using a Pyruvate Kinase Assay Kit and a LDH Assay Kit, respectively (Nanjing Jiancheng, China). Sperm pellets were lysed and centrifuged. The supernatants were used to analyze the pyruvate kinase and LDH activity according to the manufacturer's instructions.

\section{Assessment of acrosome reaction, motility and membrane integrity}

Acrosome reaction was detected by fluorescein isothiocyanate-conjugated peanut agglutinin (FITCPNA) binding [25]. The staining was monitored and photographed with an epifluorescent microscope (80i; Nikon). As shown (see online suppl. material) in Suppl. Fig. 2A, the sperm could be classified into 2 groups: acrosome reaction (blue arrow) and acrosome no-reaction (white arrow).

Sperm motility was assessed with a computer-assisted spermatozoa analysis system (CASA) (Integrated Semen Analysis System; Hview, Fuzhou, China). A minimum of 300 sperm were observed from at least five randomly selected fields. Recorded parameters were: total motility (\%), progressive motility (\%), beat-cross frequency (BCF, Hz), curvilinear velocity (VCL, $\mu \mathrm{m} / \mathrm{s}$ ), straight-line velocity (VSL, $\mu \mathrm{m} / \mathrm{s}$ ), average-path velocity (VAP, $\mu \mathrm{m} / \mathrm{s}$ ), wobble (WOB, \%) and linearity (LIN, \%).

Membrane integrity was evaluated using a LIVE/DEAD Sperm Viability Kit (Leiden the Netherlands, L7011) [25]. Briefly, sperm suspensions were stained with SYBR-14 and PI. The staining was monitored and photographed with an epifluorescent microscope (80i; Nikon). The sperm were classified into three groups (see online suppl. material, Supp. Fig. 2B): membrane integrity (white arrow), membrane slightly damage (black arrow) and membrane damage (blue arrow).

\section{Assay of Chlortetracycline fluorescence and mitochondrial membrane potentials}

According to Ded et al. [26], sperm were incubated with $750 \mu \mathrm{M}$ chlortetracycline (CTC) solution. The CTC fluorescence patterns were evaluated with an epifluorescent microscope (80i; Nikon) [27].

JC-1 Mitochondrial Membrane Potential Detection Kit (Beyotime Institute of Biotechnology, China) was used to analyze mitochondrial membrane potentials $(\Delta \Psi \mathrm{m})$ [28]. Briefly, sperm were stained with $1 \times \mathrm{JC}$ 1. Fluorescence intensity of both mitochondrial JC-1 monomers ( $\lambda$ ex $514 \mathrm{~nm}$, $\lambda$ em $529 \mathrm{~nm}$ ) and aggregates ( $\lambda$ ex $585 \mathrm{~nm}, \lambda$ em $590 \mathrm{~nm}$ ) were detected using a monochromator microplate reader (Safire II, Tecan, Switzerland). The $\Delta \Psi \mathrm{m}$ was calculated as the fluorescence ratio of red (aggregates) to green (monomers).

\section{Sperm-zona pellucida-binding assay}

According to Bromfield et al. [29], porcine ovaries were obtained from a local slaughterhouse. The oocytes were prepared from the cumulus cell-free oocytes. Twenty-five of oocytes were placed in a 50$\mu \mathrm{L}$ droplet of fertilization medium for each group. Meanwhile, frozen-thawed sperm were incubated with AMPK activators ( 2 mM AICAR, $1 \mathrm{mM}$ Met), or inhibitor (30 $\mu$ M Compound C), CM or NCM (as a control) for 3 $\mathrm{h}$. Then $50 \mu \mathrm{L}$ of the incubated sperm suspension was added into a fertilization medium droplet containing 25 oocytes, and incubated for $2 \mathrm{~h}$ at $39{ }^{\circ} \mathrm{C}$ in a humidified atmosphere saturated with $5 \% \mathrm{CO}_{2}$. Following incubation, the total number of sperm tightly bound to each of zona pellucida was counted.

\section{Statistical analysis}

All data were tested for normality and variance homogeneity prior to statistical analysis. Data were analyzed with a general mixed model (with repeated measures), multiple comparisons with Tukey test was 


\section{Cellular Physiology Cell Physiol Biochem 2018;47:2420-2431 \\ and Biochemistry Published online: July 09, $2018 \quad \begin{aligned} & \text { DOI: 10.1159/000491616 } \\ & \begin{array}{l}\text { (c) } 2018 \text { The Author(s). Published by S. Karger AG, Basel } \\ \text { www.karger.com/cpb }\end{array}\end{aligned}$ \\ Zhu et al.: AMPK is Involved in Goat Sperm Functions}

performed using SPSS version 17.0 for Windows (SPSS Inc., Chicago, IL). The treatment (activator, inhibitor, NCM and CM) was inter-subject factor while incubation time (1 h, $2 \mathrm{~h}, 3 \mathrm{~h})$ was the intrasubject factor in that model. Each functional parameter was the dependent variable. All the values are presented as mean \pm SEM. Treatments were considered statistically different from one another at $\mathrm{p}<0.05$.

\section{Results}

\section{Localization of AMPK in goat sperm}

As shown in Fig. 1A, AMPK protein was strongly expressed in entire acrosomal region and the midpiece, whereas expressed with less intensity in the flagellum. Interestingly, phosphoThr172-AMPK was mainly distributed in the midpiece, with less intensity in flagellum, but undetected in acrosome (Fig. 1B). After induction of acrosome reaction in vitro, phosphoThr172-AMPK was strongly localized in the midpiece and the head (Fig. 1C).

AMPK phosphorylation during in vitro induction of capacitation and acrosome reaction

As shown in Fig. 2A, three CTC staining patterns were detected under an epifluorescent microscope: pattern $\mathrm{F}$ (non-capacitated sperm) corresponding to an even distribution of fluorescence on sperm head either with or without a bright equatorial band, pattern B (capacitated sperm) with fluorescence in the anterior portion of the head and pattern AR (acrosome reacted sperm) with no or very weak fluorescence over the head.

To elucidate whether AMPK was indeed related to capacitation and acrosome reaction, sperm were incubated in capacitation medium (CM) or with an inducer of acrosome reaction (A23817). Incubation in CM for $3 \mathrm{~h}$ significantly increased the percentage of pattern B sperm compared to control that was incubated in NCM (Fig. 2B). However, incubation in CM with Compound $\mathrm{C}$ resulted in a significant lower percentage of pattern $\mathrm{B}$ cells, compared to the $\mathrm{CM}$ control. Moreover, percentage of Pattern F cells in CM treatment was lower than that in NCM or CM with Compound C, while it was similar between NCM and CM with Compound C treatments. Interestingly, the percentage of cells with pattern AR was similar among the three treatments. Furthermore, compared to the NCM treatment, the tyrosine phosphorylation signal of p15 $\mathrm{kDa}$ (a capacitation marker) increased in sperm after $3 \mathrm{~h}$ of incubation in $\mathrm{CM}$, whereas it decreased in sperm that were exposed to Compound $\mathrm{C}$, indicating a delay of capacitation (Fig. 2C). Similarly, after induction of acrosome reaction with $10 \mu \mathrm{M}$ A23817, the percentage of cells with Pattern AR in capacitation treatment was much higher than noncapacitation treatment. However, the capacitation treatment supplemented with Compound C decreased the Pattern AR cells (Fig. 2D).

Importantly, compared to the non-capacitation conditions, the value of AMPK activity was higher in the treatment induced capacitation, whereas was lower in Compound $\mathrm{C}$ treatment (Fig. 2E). Furthermore, during acrosome reaction, AMPK activity also significantly increased (Fig. 2E). As shown (see online suppl. material) in Suppl. Fig. 3A, the amount of AMPK was not different among the treatments during capacitation and acrosome reaction process. However, the amount of Thr172-phosphorylated AMPK significantly increased when sperm being incubated in $\mathrm{CM}$. When $30 \mu \mathrm{M}$ of Compound $\mathrm{C}$ was added to $\mathrm{CM}$, the signal of Thr172- phosphorylated AMPK reduced compared to that without Compound C (Fig. 2F and 2G). Similarly, when acrosome reaction was induced with $10 \mu$ M A23817, the intensity of Thr172-phosphorylated AMPK increased. Moreover, addition of Compound C counteracted the increase of AMPK $\alpha$ phosphorylation induced by A23817 (Fig. 2F and 2G).

In addition, the value of progressive motility in the treatment incubated in CM was significantly higher than that of incubation in NCM. However, it decreased when Compound $\mathrm{C}$ was added to the CM (see online suppl. material, Suppl. Table 1). Furthermore, as shown in (see online suppl. material) Suppl. Table 1, an increase in total motility, BCF, VCL, VSL, VAP, WOB, LIN was observed in the treatment incubated in CM. Supplementation of Compound $\mathrm{C}$ decreased those parameters. In terms of the $\Delta \Psi \mathrm{m}$, treatments of either capacitation or 


\section{Cellular Physiology Cell Physiol Biochem 2018;47:2420-2431 \begin{tabular}{l|l} 
and Biochemistry Published online: July 09, 2018 & $\begin{array}{l}\text { C } 2018 \text { The Author(s). Published by S. Karger AG, Basel } \\
\text { www.karger.com/cpb }\end{array}$
\end{tabular} \\ Zhu et al.: AMPK is Involved in Goat Sperm Functions}

acrosome reaction in vitro significantly improved the value of $\Delta \Psi \mathrm{m}$, whereas addition of Compound C significantly reduced $\Delta \Psi \mathrm{m}$ (see online suppl. material, Suppl. Fig. 4).

\section{AMPK activity affects acrosome reaction during incubation in TCG extender}

In order to reveal whether activation of AMPK stimulated acrosome reaction during incubation, sperm were incubated in TCG extender with or without $2 \mathrm{mM}$ AICAR, $1 \mathrm{mM}$ Met or $30 \mu \mathrm{M}$ Compound $\mathrm{C}$ at $37^{\circ} \mathrm{C}$. Motility, membrane integrity, acrosome reaction and $\Delta \Psi \mathrm{m}$ were detected. As shown (see online suppl. material) in Suppl. Table 2, addition of AICAR and Met significantly increased progressive motility compared to that of control, while supplementation of Compound $\mathrm{C}$ decreased it. Moreover, the values of BCF, VCL, VSL, VAP, WOB, LIN in AICAR and Met treatments were higher than that of control. On the contrary, inhibition of AMPK by Compound $\mathrm{C}$ decreased the aforementioned parameters (see online suppl. material, Suppl. Table 2). In addition, addition of AICAR or Met significantly increased membrane integrity compared to that of control, while inhibition of AMPK resulted in a reduction of membrane integrity ((see online suppl. material, Suppl. Fig. 5A).

Interestingly, activation of AMPK by AICAR or Met enhanced acrosome reaction during incubation of sperm in TCG extender. The percentage of sperm that underwent acrosome reaction (AR pattern acrosome) in AICAR and Met treatment was significantly higher than that of control. On the contrary, inhibition of AMPK by Compound C led to a decrease in the rate of sperm with $\mathrm{AR}$ pattern acrosome at $3 \mathrm{~h}$ of in vitro

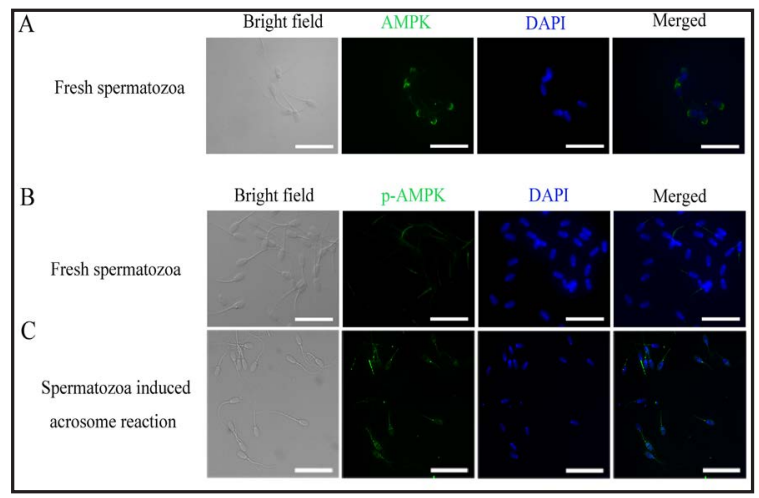

Fig. 1. Images of AMPK and AMPK phosphorylation. Immunolocalization of AMPK in fresh goat sperm (A). Immunolocalization of phospho-Thr172-AMPK in fresh goat sperm (B) and acrosome reaction-induced goat sperm (C). Bars $=30 \mu \mathrm{m}$.

Fig. 2. (A) Illustration of sperm with different fluorescence patterns. Pattern F, non-capacitated sperm corresponding to an even distribution of fluorescence in the head either with or without a bright equatorial band; Pattern B, capacitated sperm with fluorescence in the anterior portion of the head; Pattern AR, acrosome reacted sperm with no or very weak fluorescence over the head. (B) CTC assay of sperm that were induced capacitation in vitro. (C) The phosphorylated tyrosine residue of protein was detected by western blotting using anti-phosphotyrosine antibody. (D) Acrosome reaction was detected with FITC-PNA and PI staining. (E) AMPK activity was detected with a AMPK ELISA Kit. (F) Expression of AMPK and phospho-Thr172-AMPK were detected by western blotting during capacitation and acro-

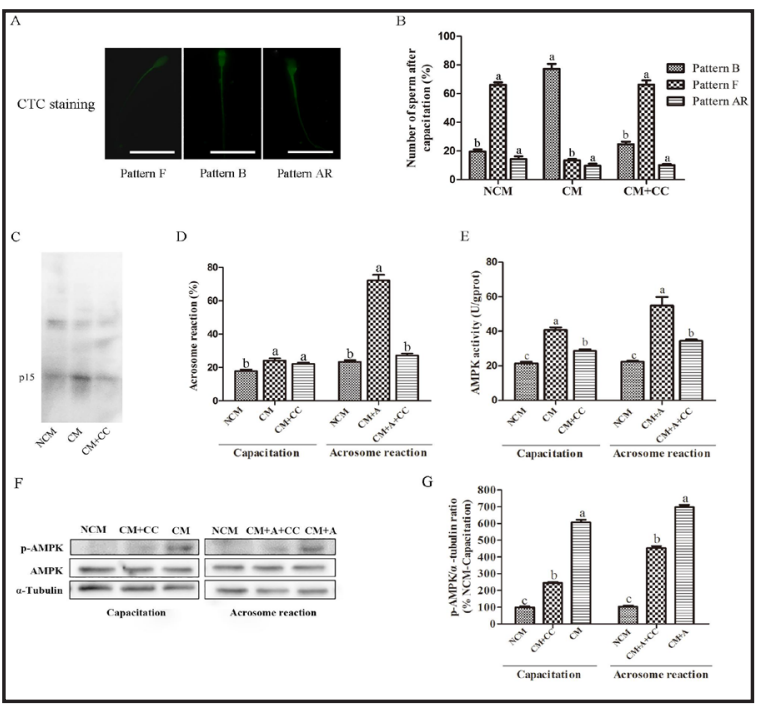
some reaction in vitro. (G) Densitometric analy-

sis of the p-AMPK signal normalized against the $\alpha$-tubulin during capacitation and acrosome reaction in vitro ( $n=3$ independent replicates). NCM, sperm incubated in non-capacitation medium; CM, sperm incubated in capacitation medium; $\mathrm{CM}+\mathrm{CC}$, sperm incubated in capacitation medium with Compound C; $\mathrm{CM}+\mathrm{A}$, sperm incubated in capacitation medium with A23817; $\mathrm{CM}+\mathrm{A}+\mathrm{CC}$, sperm incubated in capacitation medium with Compound C and A23817. Bar $=24 \mu \mathrm{m}$. 


\section{Cellular Physiology Cell Physiol Biochem 2018;47:2420-2431 \begin{tabular}{l|l} 
DOI: 10.1159/000491616 & $\begin{array}{l}\text { O 2018 The Author(s). Published by S. Karger AG, Basel } \\
\text { www.karger.com/cpb }\end{array}$
\end{tabular}

incubation, compared to the control (see online suppl. material, Suppl. Fig. 5B).

Furthermore, addition of AICAR and Met significantly increased the $\Delta \Psi \mathrm{m}$ at $1 \mathrm{~h}, 2 \mathrm{~h}$ of incubation. Addition of Compound $\mathrm{C}$ resulted in a significant reduction of $\Delta \Psi \mathrm{m}$ at $1 \mathrm{~h}$ and $3 \mathrm{~h}$ of incubation when compared to other treatments. Interestingly, supplementation of AICAR yielded the highest value among the treatments at $3 \mathrm{~h}$ of incubation (see online suppl. material, Suppl. Fig. 5C).

In order to confirm that addition of AICAR, Met or Compound C modulated activity of AMPK, AMPK activity and its phosphorylation were detected. As shown in Fig. $3 \mathrm{~A}$, exposure to AICAR and Met increased AMPK activity at $1 \mathrm{~h}, 2$ $\mathrm{h}$ and $3 \mathrm{~h}$ of incubation, whereas addition of Compound $\mathrm{C}$ decreased it $(\mathrm{p}<0.05$, Fig. $3 \mathrm{~A})$. However, the amount of AMPK was not different among the treatments during $3 \mathrm{~h}$ of incubation (see online suppl. material, Suppl. Fig. 3B). In addition, amount of phospho-Thr172-AMPK significantly increased at $1 \mathrm{~h}, 2$ $\mathrm{h}$ and $3 \mathrm{~h}$ of exposure to AICAR or Met. In contrast, exposure to Compound $\mathrm{C}$ reduced the level of phospho-Thr172-AMPK (Fig. 3B and $3 \mathrm{C}$ ). Moreover, compared to the control, addition of AICAR or Met increased the percentage of sperm that were positive for anti- phosphoThr172-AMPK IgG, whereas complementation with Compound $\mathrm{C}$ decreased the positive cells (Fig. 3D).

\section{AMPK activity affects sperm energetic metabolism}

As shown in Fig. 4A, lactate content increased significantly in the control during incubation. Addition of AICAR or Met significantly elevated the value of lactate content at each time point, when compared to control. On the contrary, supplementation of Compound C reduced it $(\mathrm{p}<0.05)$. Similarly, compared with the control, AICAR or Met significantly increased ATP content, whereas Compound $\mathrm{C}$ decreased it at each time point during incubation (Fig. 4B).

To evaluate whether the activators (AICAR or Met) or inhibitor (Compound C) regulated sperm energetic metabolism, activity of PK and LDH was detected, localization and expression of PK and LDH were also assessed by immunofluorescence and western

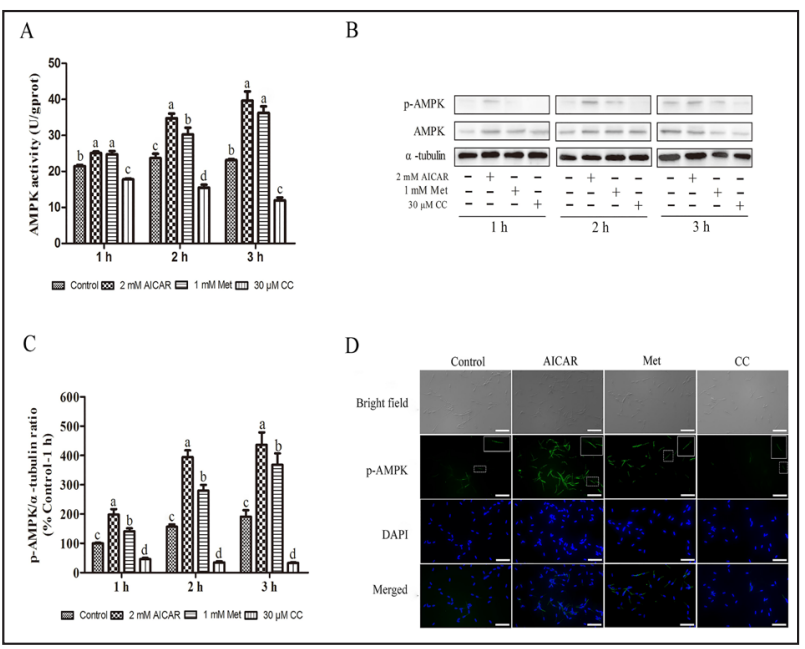

Fig. 3. (A) AMPK activity was detected with a AMPK ELISA Kit during $3 \mathrm{~h}$ of incubation. (B) The activation (AICAR and Met) or inhibition (Compound C) of AMPK were evaluated by western blot during $3 \mathrm{~h}$ of incubation. (C) Densitometric analysis of the p-AMPK signal normalized against the $\alpha$-tubulin during $3 \mathrm{~h}$ of incubation ( $\mathrm{n}=3$ independent replicates). (D) Immunofluorescent images of AMPK phosphorylation in sperm incubated with AMPK modulators. Bars $=40$ $\mu \mathrm{m}$.

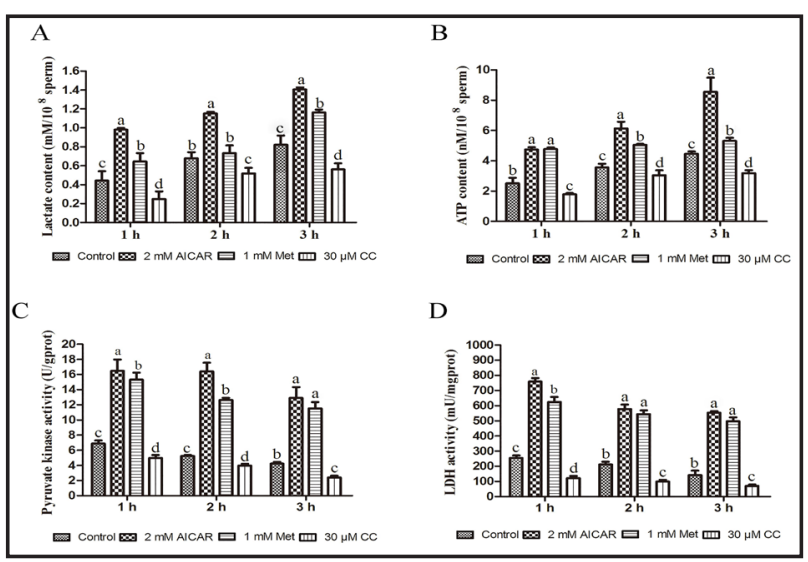

Fig. 4. Effects of AMPK modulators on sperm lactate content (A), ATP content (B), pyruvate kinase activity (C) and LDH (D) activity during $3 \mathrm{~h}$ of incubation. Bars represent mean values \pm SEM ( $n=3$ independent replicates). Different lower-case letters denote significant differences $(\mathrm{p}<0.05)$. 


\section{Cellular Physiology Cell Physiol Biochem 2018;47:2420-2431

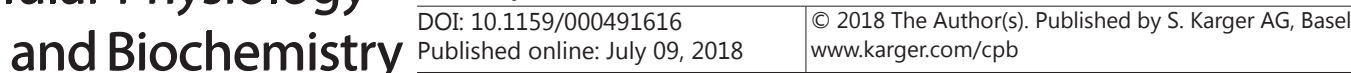 Zhu et al.: AMPK is Involved in Goat Sperm Functions}

blotting. As shown in Fig. 4C, when compared to the control, addition of AICAR or Met significantly increased PK activity, but supplementation of Compound $\mathrm{C}$ decreased the activity at each time point ( $p<0.05$, Fig. 4C). Similarly, addition of AICAR or Met significantly increased the value of $\mathrm{LDH}$ activity, while Compound $\mathrm{C}$ decreased the value (Fig. 4D). Interestingly, as shown in Fig. 5A, PK was localized in the acrosomal area and the midpiece, while LDH was expressed in the tail, strongly in midpiece (Fig. 5B).

In order to evaluate whether AMPK modulates sperm capacitation and acrosome reaction via regulating energetic metabolism, lactate content, ATP content, activity of PK and LDH were also assessed after capacitation and acrosome reaction. Compared to NCM group, capacitation in vitro led to high level of lactate in sperm. Interestingly, exposure sperm to $\mathrm{CM}$ plus Compound $\mathrm{C}$ resulted in similar

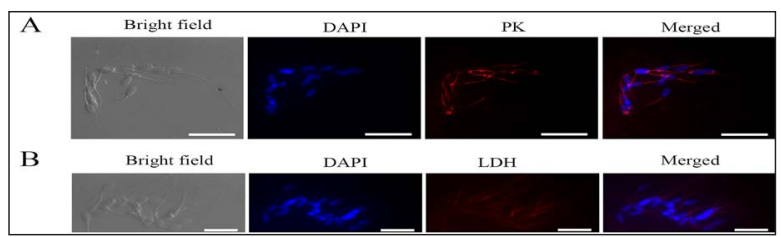

Fig. 5. Immunolocalization of pyruvate kinase (PK) (A), LDH (B) in goat sperm. Bars $=30 \mu \mathrm{m}$.

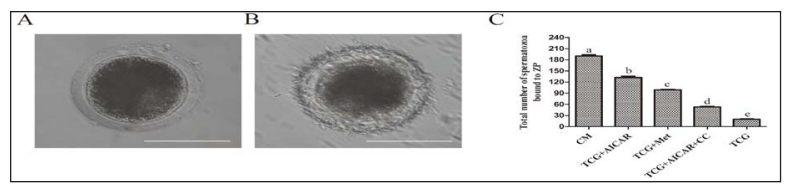

Fig. 6. Effect of AMPK modulators on sperm-zona pellucida (ZP) binding capacity. (A) zona pellucida, (B) sperm-zona pellucida complexes, $(C)$ total number of sperm bound to zona pellucida. CM: sperm incubated in capacitation medium; TCG+AICAR: sperm incubated in TCG extender with AICAR; TCG+Met: sperm incubated in TCG extender with Met; TCG+AICAR+CC: sperm incubated in TCG extender with AICAR and Compound C; TCG: sperm incubated in TCG extender without ARCAR or Met or CC. Bars represent the mean \pm SEM ( $n=3$ independent replicates, each replicate was performed 25 zona pellucidas). Bars $=100 \mu \mathrm{m}$. level of lactate to the NCM group (see online suppl. material, Suppl. Fig. 6A). Moreover, induction of acrosome reaction with A23817 yielded high content of lactate, whereas Compound C attenuated the effect of A23817 (see online suppl. material, Suppl. Fig. 6A). Similarly, ATP content significantly increased during induction of capacitation and acrosome reaction. Furthermore, addition of Compound C significantly decreased ATP content during capacitation and acrosome reaction (see online suppl. material, Suppl. Fig. 6B). Importantly, in vitro induction of capacitation and acrosome reaction also caused an increase in the activity of PK and LDH. However, AMPK inhibitor Compound C inhibited the increase in activity of these two enzymes during these processes (see online suppl. material, Suppl. Fig. 6C and 6D).

\section{AMPK activity is involved in sperm capacity to bind to zona-pellucida}

To verify the role of AMPK in goat sperm functions, a heterologous sperm-ZP binding assay was conducted. Zona pellucida and sperm-zona pellucida complexes were showed in Fig. 6A and 6B. In vitro induction of capacitation was used as a positive control. Incubation with either AICAR or Met led to high value of total number of sperm binding to heterologous ZP. However, Compound C counteracted the effect of AICAR (Fig. 6C).

\section{Discussion}

As a sensor of cell energy status, AMPK regulates metabolic pathways and simultaneously inhibits ATP-consuming anabolic pathways under different energy status or stressful conditions [7]. AMPK was expressed in the acrosome, midpiece and the entire flagellum of goat sperm, which was in consistence with that in boar [30], human [16, 17] and chicken [15] sperm. However, this observation was different to the report by Swegen et al. [31] who demonstrated that AMPK was predominantly present in the midpiece and the entire flagellum of stallion sperm. Interestingly, we found that the phospho-Thr172-AMPK was 


\section{Cellular Physiology Cell Physiol Biochem 2018;47:2420-2431 \begin{tabular}{l|l} 
DOI: 10.1159/000491616 & O 2018 The Author(s). Published by S. Karger AG, Basel \\
www.karger.com/cpb
\end{tabular} Zhu et al.: AMPK is Involved in Goat Sperm Functions}

mainly distributed in the midpiece of fresh goat sperm (Fig. 1B). However, it predominantly localized in the head and the midpiece of the sperm after in vitro induction of acrosome reaction (Fig. 1C). These observations were different with Hurtado de Llera et al. [30], who reported that phospho-Thr172-AMPK was specially present in the most apical region of the acrosome and in the equatorial subsegment of the head, and also in the midpiece of boar sperm after being incubated with TBM or TCM. Moreover, phospho-Thr172-AMPK was present in the principal piece of tail and subequatorial region in the head of stallion sperm [14, 31], mainly in the flagellum and acrosome and with a lower intensity in the midpiece of chicken sperm [15]. This contradiction reflects specific localization pattern of phospho-Thr172-AMPK in sperm from different species.

Capacitation is a series of morphological and metabolic changes that is necessary for sperm to achieve fertilizing ability [1]. In present study, we found that the value of phospho-Thr172-AMPK increased when goat sperm were exposed to capacitation medium. Interestingly, addition of AMPK inhibitor (Compound $\mathrm{C}$ ) to the capacitation medium led to reduction of AMPK activity. Under the physiological condition in vivo, acrosome reaction occurs by fusion of the cytoplasmic membrane in sperm head and the underlying outer acrosomal membrane [32], which is a necessary step for fertilization. It is well-known that calcium ionophore A23187 can induce acrosome reaction in vitro [33]. In this study, we observed that AMPK phosphorylation occurred during in vitro induction of acrosome reaction (Fig. 2F). Inhibition of AMPK activity in goat sperm incubated in the presence of Compound $\mathrm{C}$ caused a significant inhibition of acrosome reaction (Fig. 2D). Interestingly, activation of AMPK by AICAR or Met stimulated acrosome reaction during incubation in vitro. On the contrary, inhibition of AMPK by Compound C inhibited acrosome reaction (see online suppl. material, Suppl. Fig. 5B). Moreover, activation of AMPK increased total number of sperm that bound to heterologous ZP, which suggested that AMPK enhanced sperm capacitation process [20]. The data of heterologous goat sperm-porcine zona pellucida-binding assay further confirm that AMPK activity is important for sperm functions. These findings indicate that AMPK phosphorylation occurred during induction of capacitation and acrosome reaction and that AMPK activity is indeed involved in regulation of sperm capacitation and acrosome reaction. The findings obtained in this study are agreed with a previous report that AMPK inhibition reduced acrosome reaction in chicken sperm [15].

As AMPK is a sensor of cell energy status, we elucidated whether AMPK activity was related to energy metabolism during induction of capacitation and acrosome reaction in vitro. Either AICAR or Met activated AMPK, whereas Compound C inhibited AMPK phosphorylation. Interestingly, exposure goat sperm to AICAR or Met led to an increase of ATP production, while exposure to Compound C caused a decrease of ATP level (Fig. 4B), which was in line with the level of AMPK phosphorylation and AMPK activity. Therefore, AMPK activity was in 


\section{Cellular Physiology Cell Physiol Biochem 2018;47:2420-2431 and Biochemistry Published online: July 09, $2018 \quad \begin{aligned} & \text { DOI: 10.1159/000491616 } 2018 \text { The Author(s). Published by S. Karger AG, Basel } \\ & \text { www.karger.com/cpb }\end{aligned}$ \\ Zhu et al.: AMPK is Involved in Goat Sperm Functions}

accordance with ATP production during the process of in vitro capacitation and acrosome reaction. Those observations were consistent with those in chicken sperm [15] and in boar sperm [18].

In present study, we observed that phospho-Thr172-AMPK was extensively localized in the head, the midpiece and principal piece of goat sperm tail when incubated in the presence of AICAR or Met (Fig. 1C), where ATP was synthesized via mitochondrial respiration and anaerobic glycolysis [34]. PK catalyzes the final step of glycolysis, which is one of the three rateaffecting steps of the catabolic reaction cascade. This step is highly regulated and deliberately irreversible because pyruvate is a key intermediate building block for further metabolic pathways [35]. Once pyruvate synthesized, it is transformed to lactate by LDH when oxygen is absent or in short supply. Pyruvate also enters the TCA cycle for ATP production under aerobic conditions. Interestingly, we found that PK was localized in the acrosomal area and the midpiece, while LDH was expressed in the tail. The observations were in according with Feiden et al. [36] in boar sperm and Burgos et al. [37] in mouse sperm. Exposure goat sperm to AICAR or Met enhanced activity of PK and LDH, while Compound C inhibited activity of both PK and LDH (Fig. 4C and 4D). Evidences have shown that lactate, one of end-product of glycolysis, was regulated by AMPK activation in granulosa/skeletal muscle cell models [38, 39]. In this study, exposure either AICAR or Met led to sperm to enhance lactate production, while Compound $\mathrm{C}$ made sperm generate small amount of lactate, which was similar to the finding in chicken sperm [15].

In addition, $\Delta \Psi \mathrm{m}$ is strongly related with sperm fertilization [40]. AMPK have been regarded as one of the regulators of $\Delta \Psi \mathrm{m}$ in chicken [15], mouse [41], and boar [30] sperm. In present study, AMPK up-activation yielded higher $\triangle \Psi \mathrm{m}$, while AMPK inactivation reduced $\Delta \Psi \mathrm{m}$. These observations suggest that AMPK phosphorylation maintains the mitochondrial membrane potential in goat sperm. As the phospho-Thr172 is localized in the midpiece of the sperm tail, in which mitochondria are distributed, we speculate that AMPK is an essential metabolic checkpoint to stimulate sperm metabolism for maintaining $\Delta \Psi \mathrm{m}$.

Finally, motility and membrane integrity are important parameters for fertilization. We found that AMPK effectors AICAR and Met improved motility and membrane integrity, while Compound $\mathrm{C}$ decreased the two parameters during in vitro incubation. These data are agreed with those in boar sperm $[13,18]$, suggesting that AMPK activity is involved in modulating sperm motility and membrane integrity. However, Nguyen et al. [15] demonstrated that Compound $\mathrm{C}$ alone did not affect sperm membrane integrity. This contradictory observation may be due to species and semen extenders.

In conclusion, we propose a model of AMPK function (Fig. 7). Phospho-Thr172-AMPK was localized in midpiece and flagellum in fresh sperm, while mainly in the head and the midpiece of tail after the induced acrosome reaction. Induction of capacitation and acrosome reaction led to AMPK phosphorylation. Meanwhile, AMPK activity regulated sperm motility, membrane integrity, $\Delta \Psi \mathrm{m}$, capacitation and acrosome reaction via modulating lactate content, ATP production and the activities of energetic enzymes. This study for the first time provides evidence that AMPK governs goat sperm functions through energy metabolism in vitro. Therefore, modulating AMPK activity would be of benefit to develop novel strategies for preservation sperm in vitro. This finding will help to improve assisted reproductive techniques in goats and the other species.

\section{Acknowledgements}

This study was supported by the National Natural Science Foundation of China (Grant No. 31072029) for WZ. The funders had no role in study design, data collection and analysis, decision to publish, or preparation of the manuscript. We thank Dr. Bin Wu at Arizona Center for Reproductive Endocrinology and Infertility, USA, for editing the revised manuscript. Conceived and designed the experiments: ZZ, WZ. Performed the experiments: ZZ, RL and 


\section{Cellular Physiology Cell Physiol Biochem 2018;47:2420-2431 \begin{tabular}{l|l} 
DOI: 10.1159/000491616 & $\begin{array}{l}\text { O 2018 The Author(s). Published by S. Karger AG, Basel } \\
\text { www.karger.com/cpb }\end{array}$
\end{tabular} \\ Zhu et al.: AMPK is Involved in Goat Sperm Functions}

GM. Analyzed the data: ZZ, XF. Contributed reagents/materials/analysis tools: WB, YL, XF and J L. Wrote the manuscript: ZZ, WZ.

\section{Disclosure Statement}

The authors have nothing to disclose.

\section{References}

1 Gervasi MG, Visconti PE: Chang's meaning of capacitation: A molecular perspective. Mol Reprod Dev 2016;83:860-874.

2 Naz RK, Rajesh PB: Role of tyrosine phosphorylation in sperm capacitation / acrosome reaction. Reprod Biol Endocrinol 2004;2:75.

-3 Suarez SS: Control of hyperactivation in sperm. Hum Reprod Update 2008;14:647-657.

4 Yanagimachi R: The movement of golden hamster spermatozoa before and after capacitation. J Reprod Fertil 1970;23:193-196.

5 Stival C, Puga Molina Ldel C, Paudel B, Buffone MG, Visconti PE, Krapf D: Sperm capacitation and acrosome reaction in mammalian sperm. Adv Anat Embryol Cell Biol 2016;220:93-106.

6 Chang MC: The meaning of sperm capacitation. A historical perspective. J Androl 1984;5:45-50.

7 Hardie DG, Hawley SA: Amp-activated protein kinase: The energy charge hypothesis revisited. BioEssays 2001;23:1112-1119.

8 Hardie DG, Hawley SA, Scott JW: AMP-activated protein kinase-development of the energy sensor concept. J Physiol 2006;574:7-15.

-9 Momcilovic M, Hong SP, Carlson M: Mammalian TAK1 activates Snf1 protein kinase in yeast and phosphorylates amp-activated protein kinase in vitro. J Biol Chem 2006;281:25336-25343.

$>10$ Woods A, Dickerson K, Heath R, Hong SP, Momcilovic M, Johnstone SR, Carlson M, Carling D: $\mathrm{Ca}^{2+}$ / calmodulin-dependent protein kinase kinase-beta acts upstream of AMP-activated protein kinase in mammalian cells. Cell Metab 2005;2:21-33.

11 Woods A, Johnstone SR, Dickerson K, Leiper FC, Fryer LGD, Neumann D, Schlattner U, Wallimann T, Carlson M, Carling D: LKB1 is the upstream kinase in the AMP-activated protein kinase cascade. Curr Biol 2003;13:2004-2008.

12 Garcia D, Shaw RJ: Ampk: Mechanisms of cellular energy sensing and restoration of metabolic balance. Molecular cell 2017;66:789-800.

13 Hurtado de Llera A, Martin-Hidalgo D, Gil MC, Garcia-Marin LJ, Bragado MJ: AMP-activated kinase AMPK is expressed in boar spermatozoa and regulates motility. PloS one 2012;7:e38840.

14 Cordova A, Strobel P, Vallejo A, Valenzuela P, Ulloa O, Burgos RA, Menarim B, Rodriguez-Gil JE, Ratto M, Ramirez-Reveco A: Use of hypometabolic tris extenders and high cooling rate refrigeration for cryopreservation of stallion sperm: Presence and sensitivity of 5'-AMP-activated protein kinase (AMPK). Cryobiology 2014;69:473-481.

15 Nguyen TM, Alves S, Grasseau I, Metayer-Coustard S, Praud C, Froment P, Blesbois E: Central role of 5'-AMPactivated protein kinase in chicken sperm functions. Biol Reprod 2014;91:121.

-16 Calle-Guisado V, de Llera AH, Martin-Hidalgo D, Mijares J, Gil MC, Alvarez IS, Bragado MJ, Garcia-Marin LJ: AMP-activated kinase in human spermatozoa: Identification, intracellular localization, and key function in the regulation of sperm motility. Asian J Androl 2017;19:707-714.

17 Shabani Nashtaei M, Amidi F, Sedighi Gilani MA, Aleyasin A, Bakhshalizadeh S, Naji M, Nekoonam S: Protective features of resveratrol on human spermatozoa cryopreservation may be mediated through 5' AMP-activated protein kinase activation. Andrology 2017;5:313-326.

-18 Hurtado de Llera A, Martin-Hidalgo D, Gil MC, Garcia-Marin LJ, Bragado MJ: AMPK up-activation reduces motility and regulates other functions of boar spermatozoa. Mol Hum Reprod 2015;21:31-45.

19 Nguyen TM, Seigneurin F, Froment P, Combarnous Y, Blesbois E: The 5'-AMP-activated protein kinase (AMPK) is involved in the augmentation of antioxidant defenses in cryopreserved chicken sperm. PloS one 2015;10:e0134420. 


\section{Cellular Physiology Cell Physiol Biochem 2018;47:2420-2431 \begin{tabular}{l|l} 
and Biochemistry Published 10.1159/000491616 & $\begin{array}{l}\text { (c) } 2018 \text { The Author(s). Published by S. Karger AG, Basel } \\
\text { www.karger.com/cpb }\end{array}$ \\
\hline
\end{tabular} \\ Zhu et al.: AMPK is Involved in Goat Sperm Functions}

-20 Nguyen TM, Duittoz A, Praud C, Combarnous Y, Blesbois E: Calcium channels in chicken sperm regulate motility and the acrosome reaction. FEBS J 2016;283:1902-1920.

-21 Martin-Hidalgo D, Hurtado de Llera A, Yeste M, Cruz Gil M, Bragado MJ, Garcia-Marin LJ: Adenosine monophosphate-activated kinase, AMPK, is involved in the maintenance of the quality of extended boar semen during long-term storage. Theriogenology 2013;80:285-294.

22 Scott MA: A glimpse at sperm function in vivo: sperm transport and epithelial interaction in the female reproductive tract. Anim Reprod Sci 2000;60-61:337-348.

-23 Moce E, Tomas C, Blanch E, Graham JK: Effect of cholesterol-loaded cyclodextrins on bull and goat sperm processed with fast or slow cryopreservation protocols. Animal 2014;8:771-776.

-24 Salmon VM, Leclerc P, Bailey JL: Cholesterol-loaded cyclodextrin increases the cholesterol content of goat sperm to improve cold and osmotic resistance and maintain sperm function after cryopreservation. Biol Reprod 2016;94:85.

25 Zhu Z, Fan X, Lv Y, Zhang N, Fan C, Zhang P, Zeng W: Vitamin e analogue improves rabbit sperm quality during the process of cryopreservation through its antioxidative action. PLoS One 2015;10:e0145383.

26 Ded L, Dostalova P, Dorosh A, Dvorakova-Hortova K, Peknicova J: Effect of estrogens on boar sperm capacitation in vitro. Reprod Biol Endocrinol 2010;8:87.

27 Wang HF, Chang M, Peng TT, Yang Y, Li N, Luo T, Cheng YM, Zhou MZ, Zeng XH, Zheng LP: Exposure to Cadmium Impairs Sperm Functions by Reducing CatSper in Mice. Cell Physiol Biochem 2017;42:44-54.

28 Chen K, Zhang Q, Wang J, Liu F, Mi M, Xu H, Chen F, Zeng K: Taurine protects transformed rat retinal ganglion cells from hypoxia-induced apoptosis by preventing mitochondrial dysfunction. Brain Res 2009;1279:131-138.

-29 Bromfield EG, Aitken RJ, Gibb Z, Lambourne SR, Nixon B: Capacitation in the presence of methyl-betacyclodextrin results in enhanced zona pellucida-binding ability of stallion spermatozoa. Reproduction 2014;147:153-166.

30 Hurtado de Llera A, Martin-Hidalgo D, Rodriguez-Gil JE, Gil MC, Garcia-Marin LJ, Bragado MJ: Amp-activated kinase, ampk, is involved in the maintenance of plasma membrane organization in boar spermatozoa. Biochim Biophys Acta 2013;1828:2143-2151.

-31 Swegen A, Lambourne SR, Aitken RJ, Gibb Z: Rosiglitazone improves stallion sperm motility, ATP content, and mitochondrial function. Biol Reprod 2016;95:107.

32 Okamura F, Nishiyama H: The passage of spermatozoa through the vitelline membrane in the domestic fowl, gallus gallus. Cell Tissue Res 1978;188:497-508.

33 Tateno H, Krapf D, Hino T, Sanchez-Cardenas C, Darszon A, Yanagimachi R, Visconti PE: Ca ${ }^{2+}$ ionophore A23187 can make mouse spermatozoa capable of fertilizing in vitro without activation of cAMP-dependent phosphorylation pathways. Proc Natl Acad Sci U S A 2013;110:18543-18548.

34 du Plessis SS, Agarwal A, Mohanty G, van der Linde M: Oxidative phosphorylation versus glycolysis: What fuel do spermatozoa use? Asian J Androl 2015;17:230-235.

-35 Israelsen WJ, Vander Heiden MG: Pyruvate kinase: Function, regulation and role in cancer. Semin Cell Dev Biol 2015;43:43-51.

-36 Feiden S, Stypa H, Wolfrum U, Wegener G, Kamp G: A novel pyruvate kinase (PK-S) from boar spermatozoa is localized at the fibrous sheath and the acrosome. Reproduction 2007;134:81-95.

37 Burgos C, Maldonado C, Gerez de Burgos NM, Aoki A, Blanco A: Intracellular localization of the testicular and sperm-specific lactate dehydrogenase isozyme C4 in mice. Biol Reprod 1995;53:84-92.

-38 Merrill GF, Kurth EJ, Hardie DG, Winder WW: Aica riboside increases amp-activated protein kinase, fatty acid oxidation, and glucose uptake in rat muscle. Am J Physiol 1997;273:E1107-1112.

-39 Richardson MC, Ingamells S, Simonis CD, Cameron IT, Sreekumar R, Vijendren A, Sellahewa L, Coakley $\mathrm{S}$, Byrne CD: Stimulation of lactate production in human granulosa cells by metformin and potential involvement of adenosine 5' monophosphate-activated protein kinase. J Clin Endocrinol Metab 2009;94:670-677.

40 Amaral A, Lourenco B, Marques M, Ramalho-Santos J: Mitochondria functionality and sperm quality. Reproduction 2013;146:R163-174.

41 Tartarin P, Guibert E, Toure A, Ouiste C, Leclerc J, Sanz N, Briere S, Dacheux JL, Delaleu B, McNeilly JR, McNeilly AS, Brillard JP, Dupont J, Foretz M, Viollet B, Froment P: Inactivation of AMPK $\alpha 1$ induces asthenozoospermia and alters spermatozoa morphology. Endocrinology 2012;153:3468-3481. 